erratum

\title{
To ban or not to ban?
}

Clinical geneticists' views on the regulation of direct-to-consumer genetic testing

\section{Heidi Carmen Howard \& Pascal Borry}

doi:10.1038/embor.2012.141

Correction to: $E M B O \operatorname{Rep}(2012)$ 13, 791-794. doi:10.1038/embor.2012.114

This article, published in the September issue, should have been published with an accompanying supplementary information file online, containing details of the methods used.

The supplementary information file has now been published online; we apologize for the omission. 